All aspects of the transfer process were examined and adapted where necessary, from screening questions at point of referral, minimising equipment, and communication using 2 way radios. Simulation and feedback provided the opportunity to assess if these interventions were practical and could ensure a safe transfer.

\section{THE IMPACT OF COVID-19 ON THE GOSH NEWBORN SCREENING SERVICE}

Tejswurree Ramgoolam. Great Ormond Street Hospital

10.1136/archdischild-2020-gosh.102

The outbreak of COVID-19 stalled most clinical and non-clinical services across the country, however the Newborn bloodspot screening (NBS) was rated a 'critical service' because the early detection and treatment of 'at-risk' babies helps to reduce mortality and morbidity. NBS is a national public health programme where babies are screened in the first week of life for 9 conditions; 6 Inherited Metabolic Diseases (IMDs), Congenital Hypothyroidism, Cystic Fibrosis and Sickle Cell Disease. The NBS laboratory at GOSH is the largest in the UK, screening approximately 125,000 babies yearly.

Since the start of the pandemic, the NBS service has witnessed many challenges but the team always responded with remarkable resilience and flexibility. Our staffing level was reduced by $21 \%$ due to shielding regulations imposed during lockdown. Apart from donning masks and other forms of PPE, COVID-19 forced the laboratory to embrace safer and more sustainable ways of service delivery while ensuring the wellbeing of its staff. We piloted remote working across all laboratory processes and conducted risk assessments to mitigate against any impending risks while actualising established failsafe.

As a pioneer for R\&D, GOSH NBS laboratory was fully engaged in activities to support the SCID pilot (a rare condition of the immune system) before the first COVID-19 wave struck. The project discussions continued virtually and our laboratory is now set to commence the SCID pilot following the initial postponement.

The GOSH Newborn screening laboratory has delivered an uninterrupted service with no delays in the reporting of positive results including reviewing of IMD results at weekends. Our laboratory processes have been adapted and some members of our team have completed training as Peer Support Workers to enable participation in early wellbeing discussions among their colleagues. This has provided us with much needed resilience in the likely emergence of a second-wave.

\section{GREAT ORMOND STREET HOSPITAL DIGITAL GRAND ROUNDS}

Shuang Liu, Justin Poisson, Marissa Willock, Olivia Wheeler, Jonathan Smith. PGME, Great Ormond Street Hospital

\subsection{6/archdischild-2020-gosh.103}

Background Previously attendance for face -to- face Grand Round (GR) at GOSH was 20-50. Numbers were limited by location (offsite from the main hospital), publicising the events, and perceived relevance to staff.
Methods We introduced our first digital Grand Round on 28th April 2020 as Zoom based webinars. The topics were carefully chosen to cover a wide range of subjects related to COVID-19, and we were fortunate to have speakers internally who were experts in the fields. The $\mathrm{Q}$ and $\mathrm{A}$ session was via Slido and moderated by the GR chair. Diary invites were sent to all GOSH staff in addition to the previous advertising strategies: emails and screen savers. All GRs were recorded and published on the GLA YouTube channel with consent.

Results The digital GR was met with substantial enthusiasm by GOSH staff, the attendance for the first four sessions was: 290, 501,397 and 203. It was then between 85 and 147 in June. We did notice a considerable drop once trust email became unavailable on personal mobiles. From July, the attendance was around $35-70$. A steady increase in online viewing via GLA YouTube has been observed, with 400 views for one GR. In addition an international speaker was able to present online.

Discussion We were forced into hosting GR on a digital platform, but soon realised it could be a positive transformation in delivering trust-wide education. It has removed the geographic barrier so international presenters can attend. It does come with challenges such as maintaining interaction; the moderator's value for digital sessions is paramount. Furthermore, we should always actively look for new topics for continuous staff teaching engagement and ensure teaching information is widely accessible but secure.

\section{SIMULATION TO SUPPORT SYSTEM SAFETY IN A TERTIARY PAEDIATRIC HOSPITAL}

Emma Broughton. Great Ormond Street Hospital

\subsection{6/archdischild-2020-gosh.104}

Background The Clinical Simulation Centre (CSC) at GOSH, now provide one of the largest in-situ simulation programmes in the region. Prioritising advancement of the patient safety agenda, we work closely with our Quality and Safety teams to embed key safety themes within out trust wide in- situ curriculum. A key objective of delivering simulation in-situ in the clinical setting; is to identify and mitigate against hidden risks or 'latent safety threats' in the clinical environment; which could cause unintended harm to patients or staff.

Method Over the past year, the CSC team have begun to expand the applications of the pan-trust in-situ programme to deliver a number of Systems Safety exercises. These simulations focus purely on rehearsal and refinement of process and systems, towards uncovering and mitigating against latent safety threats or gaps in practice. A reporting tool has been developed by the team; to capture risks /identify mitigating actions.

Results In recent months, the Covid-19 pandemic presented our teams with many new or unfamiliar working practices. This context further shifted our focus towards Systems Safety Simulations; with the aim of enabling our clinical teams to focus on rehearsing and preparing for new ways of working. These exercises delivered in partnership with clinical teams across the trust; successfully supported the development of 5 new clinical guidelines during the pandemic. In one exercise alone; 11 latent safety threats (LSTs) were captured and managed with the appropriate teams. LST themes included; availability of resources |Communication and co-ordination in PPE 